\title{
Jin-Ho Park $\mid$ Analysis and Synthesis in Architectural Designs: A Study in Symmetry
}

Jin-Ho Park describes and demonstrates methods of analysis and synthesis in architectural composition, based on the algebraic structure of the symmetry groups of a regular polygon. The Popenoe House by Rudolf Schindler is analyzed to show a unique application of symmetry operations with regard to the spatial organization. A similar geometric order and symmetric technique is applied to the generation of a new design.

\section{Introduction}

Ordered designs are frequently encountered in art and architecture. The underlying structure of their spatial logic may be discussed with regard to the use of symmetry principles in mathematics [Weyl 1952]. In architectural designs, the use of symmetry may sometimes be apparent immediately by just looking at designs, although the final design is seemingly asymmetrical; or various symmetries are manifested in the parts of the designs, yet not immediately recognizable despite an almost obsessive concern for symmetry. At this point, it is crucial to develop a formal methodology, which may clearly elucidate different hierarchical levels of the use of symmetry in architectural designs. In an effort to do this, before proceeding to analytic and synthetic applications, we discuss a methodology founded on the algebraic structure of the symmetry group of a regular polygon in mathematics. The approach shows how various types of symmetry are superimposed in individual designs, and illustrates how symmetry may be employed strategically in the design process. Analytically, by viewing architectural designs in this way, symmetry, which is superimposed in several layers in a design, becomes transparent. Synthetically, architects can benefit from being conscious of using group operations and spatial transformations associated with symmetry in compositional and thematic development. The advantage of operating with symmetry concepts in this way is to provide architects with an explicit method not only for the understanding of symmetrical structures of sophisticated designs, but also to give architects insights for the construction of new designs by using symmetry operations. The objective of this paper resides in searching out the fundamental principles of architectural composition. A study of the fundamental principles of spatial forms in architecture is an essential prerequisite to the wider understanding of complex designs as well as the creation of new architectural forms. There are many ideas about what the fundamental principles of form are. I stand by Goethe's theory of metamorphosis in The Metamorphosis of Plants [Goethe 1946]. His theory centered on the notion that there may be an ideal form or urform, key to understanding the subsequent development of forms. Based on the urform, a variety of new designs can be developed. Later on, Gottfried Semper used the term "tectonics" to describe the theoretical viewpoint of the universal principles of forms in nature and in art, writing, "Tectonics is an art that takes nature as a model - not nature's concrete phenomena but the uniformity [Gesetzlichkeit] and the rules by which she exists and creates" [Hermann 1989: 219]. He was convinced that the understanding of fundamental principles would aid the generation of new creative work through modification, transformation and development. In his article "In the Cause of Architecture: Composition as Method in Creation”, Frank Lloyd Wright laid emphasis on the study of the principles of composition, claiming that "geometry [is] at the center of every 
Nature-form we see" [Wright 1928]. By looking into nature and grasping the fundamental principles at work, architectural forms that are not imitative but creative can be developed. In this paper, I substantially recount the methodology employed in my previous papers [Park 1995; 2000] and then create a new design in order to demonstrate the application of the method in formal composition. This paper is composed of the following three parts:

1. An outline of the important properties of the symmetries of regular polygons;

2. An analysis of an existing architectural design;

3. The synthesis of an abstract design with respect to the symmetries associated with the square.

\section{The methodology of symmetry}

Symmetry operations are concerned with spatial displacements or motions that take a shape and move it in such a way that all the elements of the shape precisely overlay one another, so that, despite the displacement, the shape appears not to have moved from its original position. For example, if we rotate a square $90^{\circ}$ around its center, it appears unchanged, whereas if we rotate an equilateral triangle $90^{\circ}$ around its center, it would appear to have changed its position. The motion of rotation through $90^{\circ}$ is called a symmetry operation of the square; it is not a symmetry of the triangle. Mathematicians call the collection of all the symmetry operations or motions that leave a particular geometric object fixed its symmetry group. The symmetry group of a twodimensional design can be finite or infinite. Infinite groups are the symmetries of infinite patterns such as tilings or infinite wallpaper patterns. Infinite symmetry groups include the motion of translation, which is a shifting of the entire pattern one unit (think of moving an infinite tiling of squares one unit; each square would lie on its neighbor; the appearance of the tiling would be unchanged.) Point groups are finite symmetry groups and correspond to a finite design, such as a single square. Only eight operations can be performed on the square and leave it occupying the same position in space: four rotations through the point at its center and reflections in four lines through this point. Let us provide an elementary account of the mathematical structure of finite symmetry groups in particular, the point groups in two dimensions. There are two types of finite point groups: the dihedral groups denoted by $\mathrm{D}_{n}$ for some integer $n$; and the cyclic groups denoted by $\mathrm{C}_{n}$. The spatial transformations of a dihedral group comprise rotation and mirror reflection; the cyclic group contains rotation only. The point groups have no translation. The number of symmetry operations in a finite group is called its order. The symmetry group $\mathrm{D}_{n}$ has $2 n$ elements or operations, while $\mathrm{C}_{n}$ has $n$ elements, and so is of order $n$. For example, the symmetry group of the square is the dihedral group $\mathrm{D}_{4}$ of order 8 . The eight distinguishable spatial transformations that comprise this group are four quarter-turns and four reflections, one each about the horizontal and vertical axes and the leading and trailing diagonal axes. $\mathrm{C}_{4}$ has four spatial transformations: the four quarter-turns. It is the symmetry group of an object like a pinwheel, which has rotational but not mirror symmetry. Since every symmetry operation in c 4 is included in $\mathrm{D}_{4}$, mathematicians say $\mathrm{C}_{4}$ is a subgroup of $\mathrm{D}_{4}$. And in fact $\mathrm{C}_{n}$ is a subgroup of $\mathrm{D}_{n}$ for any $n$. We can diagram the relationship between the different subgroups of symmetries of a square (Figure 1). In the diagram, we color the square in ways that destroy some of its symmetries. Figure 1 illustrates all possible subgroups of symmetries; the dark square has the full symmetry group $\mathrm{D}_{4}$ with 8 symmetry operations. The three squares in the second row each have four elements in their symmetry group. For example, the first square has reflection in vertical and

86 NEXUS NETWORK JOURNAL - VOL. 3, NO. 1, 2001 
horizontal mirrors, and rotation through $180^{\circ}$ and $360^{\circ}$. The second square in this row, with the pinwheel design, has symmetry group $\mathrm{C}_{4}$ of rotations through $90^{\circ}, 180^{\circ}, 270^{\circ}$ and $360^{\circ}$ degrees.

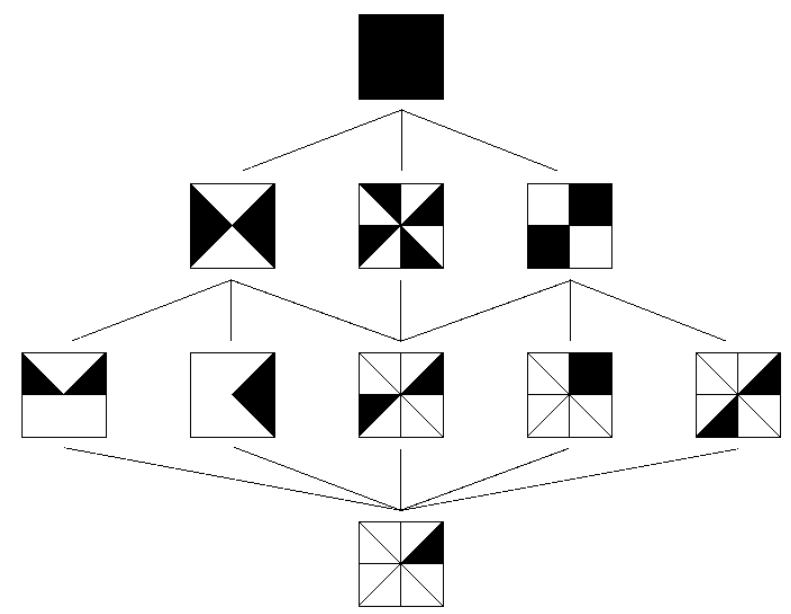

Figure 1. The lattice of subsymmetries of the square: at the top is D4 of order 8, below are subsymmetries of order 4 , then below that again of order 2 , and finally $\mathrm{c} 1$ of order one, the unit element

The symmetry groups of the square in the third row each have two elements, while the square in the bottom row has only the identity motion in its symmetry group, that is, no rotation less than the full turn through $360^{\circ}$. The structure of the diagram can be accounted for in two ways: from top to bottom, symmetries are "subtracted" from the full symmetry of the square; conversely, from the bottom to the top, subsymmetries are "added" to achieve higher orders of symmetry. These two opposing but complementary ways of using the diagram support our approach to the subsymmetry analysis and synthesis of architectural design.
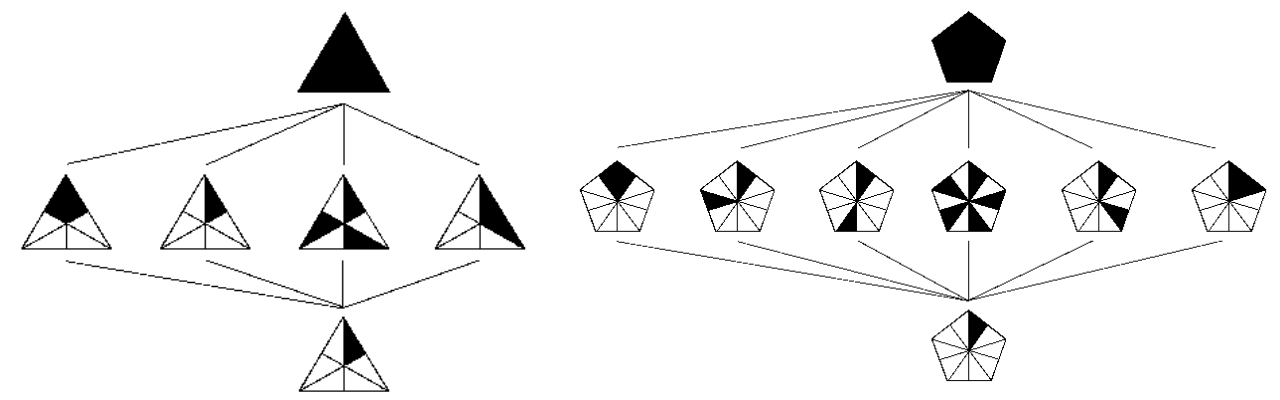

Figure 2.The lattice of subsymmetries of an equilateral triangle (left) and a regular pentagon (right)

The lattices of subsymmetries of other polygons such as an equilateral triangle, pentagon, etc. can be considered as well in this hierarchical order as shown in Figure 2. A polygon with $n$ edges has at most dihedral symmetry of order $2 n$, where the order of a finite group is the number of 
elements, here the number of symmetry operations. The subgroups of the symmetry group of a regular $n$-gon are ordered in the lattice diagram as shown in Figure 3 . For instance, $\mathrm{D}_{3}$ is the group of symmetries of an equilateral triangle, which has order 6 with its $\mathrm{D}_{1}, \mathrm{C}_{3}$ and $\mathrm{C}_{1}$ subsymmetries. Furthermore, we can generalize the lattice diagram of the regular polygon, which shows its hierarchical order of subsymmetries (Figure 3).<smiles>[CH]1CC2[CH]C12</smiles><smiles>ClC1[C@H]2[CH][C@H]1[C@@H]2Cl</smiles><smiles>[C]1[CH][CH]1</smiles>
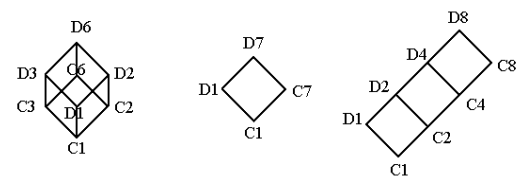

Figure 3. The lattice of subsymmetries of regular polygons: equilateral triangle, square, pantagon, hexagon, heptagon, and octagon

\section{Composition in the architecture of Rudolf Michael Schindler}

Schindler used proportion and symmetry to govern the spatial organization of his architecture. His proportional method was explicitly described in his article, "Reference Frames in Space" [Schindler 1946]. ${ }^{1}$ Schindler chose a 48 -inch (4-foot) increment as his unit module. Based on this simple computation of the given module together with its multiples and fractions $(1 / 2,1 / 3$, and 1/4), it was possible for him to derive all necessary architectural dimensions not only for major rooms but also for minor details, including doors and furniture. Using such a proportional system, architects can think, calculate and draw architectural space without mechanical tools. ${ }^{2}$ Shindler's debt to symmetry, particularly the hybrid use of various subsymmetries in a single project, is astonishing. Although he never wrote any article on symmetry, symmetries are deeply imbedded in his designs throughout his career. Whereas Schindler's early designs rely mainly on rigorous orthogonal planning, his later works seem to deny any consistent principles of symmetry. However, deeper investigation reveals that this is not so; for example, in his later works, he increasingly exploited the diagonal axis. The application typifies the "butterfly patterns: a design, which split into two wings with certain angles along the main axis". ${ }^{3}$ The interweaving of ideas of proportion and symmetry is one of the major compositional tools throughout Schindler's career [Park 1999]. The Free Public Library Project (1920) and the How House (1925) stand out from Schindler's other work during this period in their conscious play around the diagonal axis and a 48-inch unit system. Whereas the Library project is composed through the superimposition of a variety of subsymmetries without using the pure rotational subsymmetries such as $\mathrm{C}_{4}$ and $\mathrm{C}_{2}$, the How House is mainly determined by reflective symmetry along a diagonal axis 6 (Figure 4). ${ }^{4}$

Later on, in the Schindler Shelter, he utilizes symmetry operations, in particular rotation and reflection, to generate plan prototypes as well as their variations. ${ }^{5}$ In the analysis that follows, we isolate individual elements of the design that rely on a symmetrical order to identify the local and overall symmetry of the project. Whereas in most cases the whole design is seemingly asymmetrical, despite an almost obsessive concern for symmetry in its individual parts, the analysis demonstrates how various symmetry operations are involved in each of the parts of the design, revealing the underlying structure of its spatial order. Thus, the architect's use of symmetry principles in the design becomes more evident. 

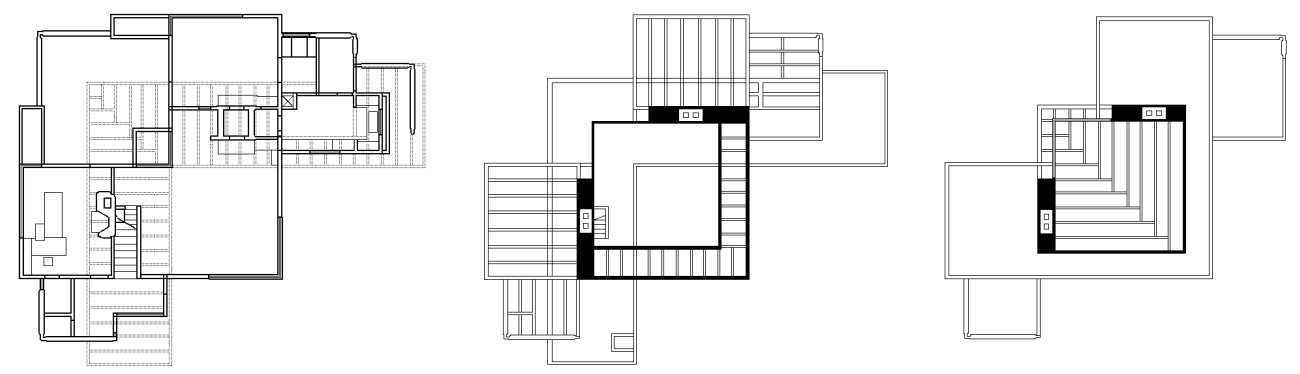

Figure 4. R.M. Schindler, How House, 1925. The overall floor plan and ceiling structure are set along the diagonal axis that forms the butterfly pattern. Left, gallery level including porch over terrace; middle, ceiling over dining room and study with aisle extensions into the corner living room; right, ceiling structure over living room

\section{The analytic example}

In this section, we take an architectural project that appears to use symmetry and scrutinize the plan to discover its underlying symmetry structure. Schindler's Popenoe House, designed in 1922 but later demolished, is the subject of our analysis. To begin the analysis, I examined the archival materials on the house, including sketches, drawings, details and photographs, housed at the Architectural Drawing Collection, University of California, Santa Barbara. I then enhanced them through the reconstruction of new drawings. This research process is essential to understand the formal and spatial aspects of the work, to clarify Schindler's design ideas, and to evince the application of his methodology in formal composition. ${ }^{6}$
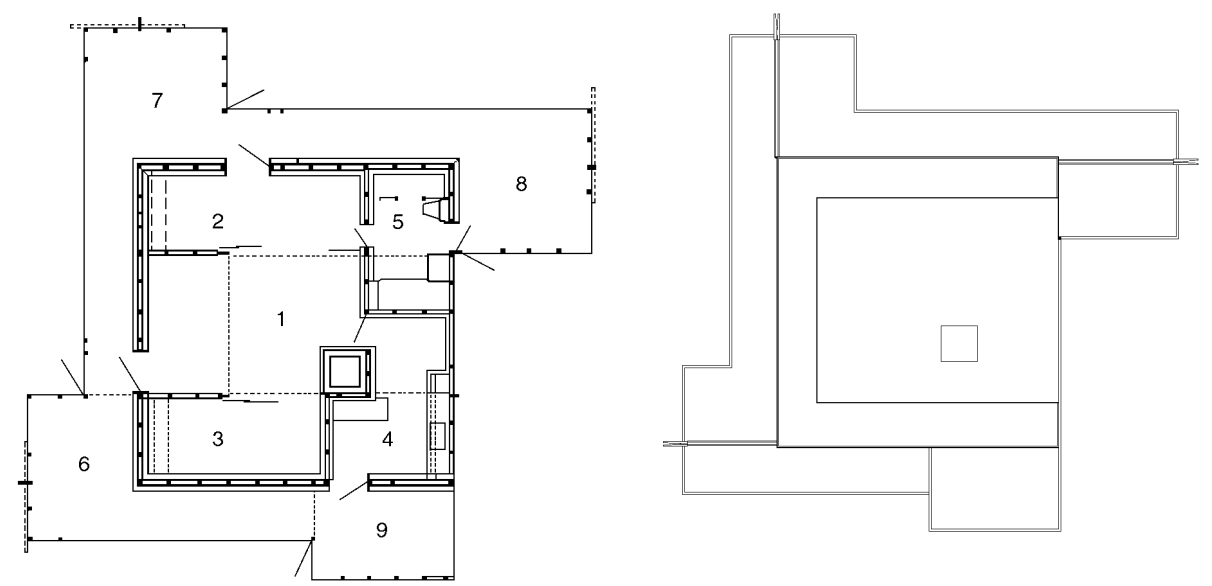

Figure 5. On the left, first floor plan of the final design of the Popenoe cabin (1. living room; 2. Her Room; 3. His Room; 4. Kitchen; 5. Bathroom; 6. Dining Porch; 7. Living Room; 8. Sleeping Porch; 9. Kitchen Porch). On the right, roof plan 
The single-story desert cabin was planned for Paul Popenoe at Coachella, California. It is one of three cabins (the cabin for Popenoe, the cabin for Philip Lovell, and the Carton Park House) that Schindler developed during the period. The basic character of the designs derives from that of the Kings Road House (1921-2) where "a marriage between the solid permanent cave and the open lightweight tent" is a key feature [Gebhard 1980; 52]. ${ }^{7}$ In fact, the three cabins have in common their designs and the use of materials (in Gebhard's words, "the cave-tent shelter of concrete, wood and canvas"), which relate the projects to the climate, region and the surroundings. ${ }^{8}$ The Popenoe cabin is a single-room type (Figure 5). The living room, located in the center of the house, is surrounded by various activity areas, including kitchen, bedrooms and utility room. The rooms are divided by thin internal walls and sliding doors, providing spatial flow and flexibility within a minimum area and optimizing the use of interior space. Four porches, each with its own entrance door, are situated in a spiral manner around the main body of the house, providing intermediate spaces for an interaction between interior and exterior. The design on a rectangular site is extremely simple, both structurally and spatially. However, in its transparent interplay of forms, it is one of the most striking examples of Schindler's lifelong use of proportions and symmetry to govern spatial organization. The fundamental 48-inch unit system and symmetry operations determine all the major decisions of the spatial organization as well as the architectural details. The primary layout of the house lies within a 22-foot by 22 -foot square, over which is laid a 48-inch module grid system. The sides of the square are subdivided into 6-foot, 10-foot and 6-foot segments, concentrically, which produces an A-B-A rhythm of the parts that relate to each other as 3:5:3. The concentric spatial schema forms an absolute four-fold symmetry with the square, invoking a classical design vocabulary. ${ }^{9}$ The parti, or underlying geometrical scheme, is a tool that governs spaces with regularity and shows the extreme clarity of its geometrical origin (Figure 6).
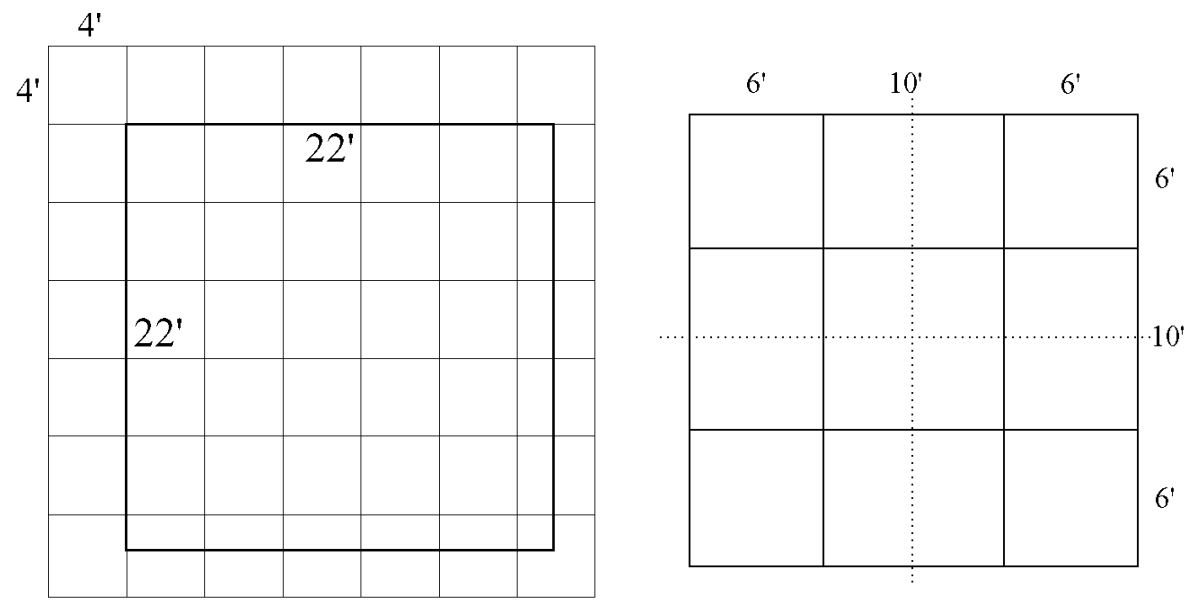

Figure 6. The basic parti of the plan. Left, basic parti with the unit system; right, the $\mathrm{D}_{4}$ subdivision of the parti

Based on the parti, the major spaces of the house are juxtaposed. The seemingly random spatial arrangement of the final design is in contrast with the complete geometric regularity of the parti, and its overall spatial configuration is asymmetric. Four additional screened porches, indicated on the plan as Living, Sleeping, Kitchen and Dining, connected by corridors, are disposed in the

90 NEXUS NETWORK JOURNAL - VOL. 3, NO. 1, 2001 
pinwheel type of $\mathrm{C}_{4}$ symmetry around the central square plan. These wrap around the primary square. The four porches and corridors are designed so that they can be closed sometimes. The length of the porch wings increase in increments of 3 feet, 4 feet, 6 feet, and 10 feet as one moves clock-wise around the house. The entire floor plan forms a spiral shape, which reinforces the impression of rotation. Whereas the basic composition of the four porches seems to derive from the strict pinwheel type of $\mathrm{C}_{4}$ symmetry, the final design is not absolute (Figure 7).
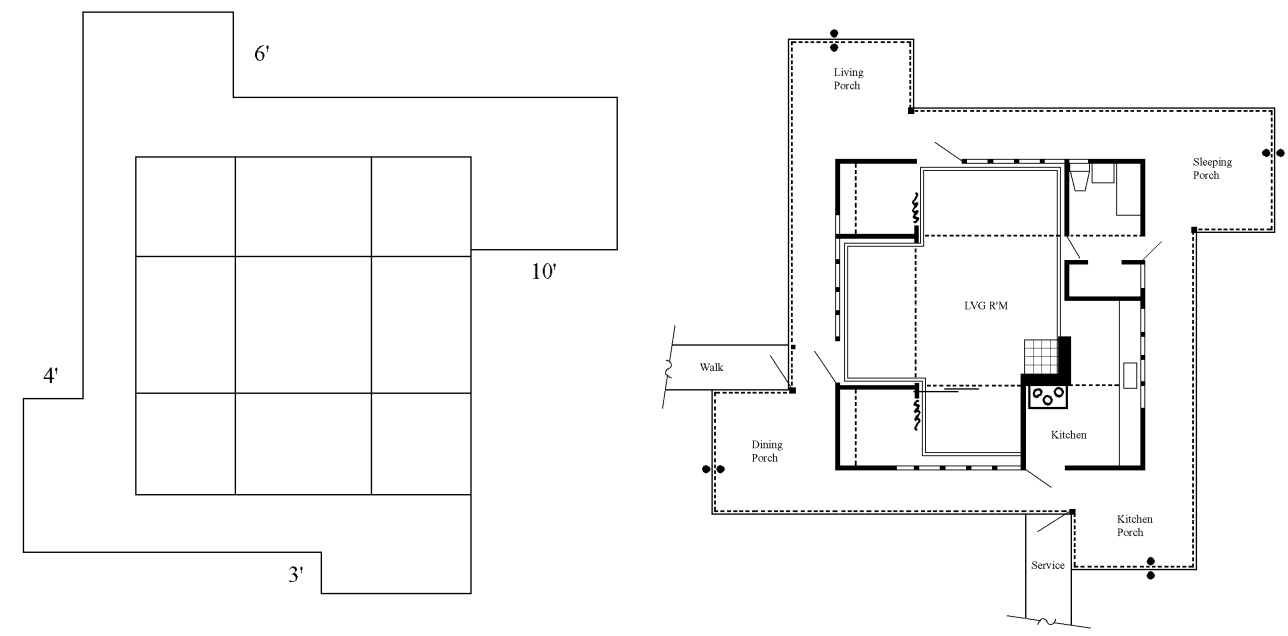

Figure 7. Left, basic parti with the pinwheel type of $\mathrm{C}_{4}$ symmetry; right, the initial scheme for the cabin, date July 1922

The architect's original idea for the composition is clearly shown in Figure $7 \mathrm{~b}$, where four porches, including the corridors, entrance doors and window openings, are determined by $\mathrm{C}_{4}$ cyclic symmetry. Thus we see how the asymmetric final design derives from a disciplined understanding of the principle of rotational symmetry and is not merely arbitrary. ${ }^{10}$ The fireplace is set along the $\mathrm{D}_{1}$ diagonal axis (Figure 8).

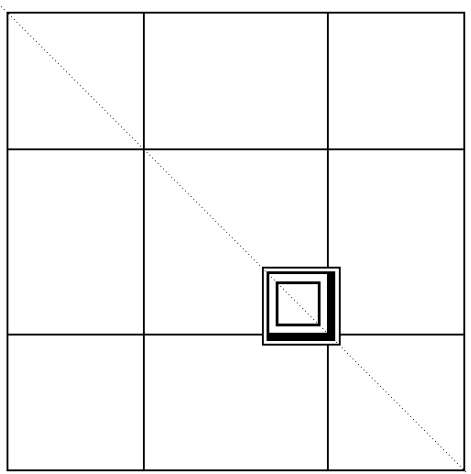

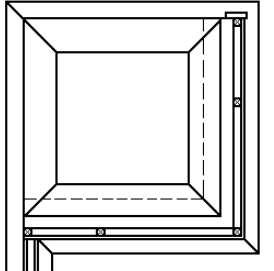

2' high

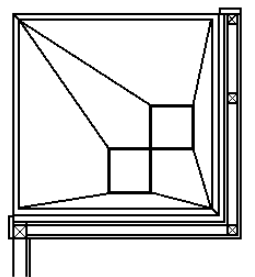

$9^{\prime}$ high

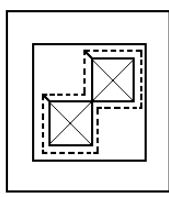

a roof plan

Figure 8. a, left) the fireplace set along the diagonal axis; b, right) the fireplace horizontal sections cut 2 feet and 9 feet above the level of the rloor, and a roof plan 
This diagram in Figure 8a illustrates that the diagonal of the square defines the axis of the living space. The fireplace could have been located on any side in the living room, yet Schindler has chosen to place it in the corner of the living area. The diagonal setting provides the dynamic sense of spatial depth and spatial experience of openness. In addition, the details of the fireplace reiterate the architect's use of the diagonal symmetry as shown in Figure 8b. The symmetric possibilities of the design are further evidenced by the arrangement of the ceiling structure. It reinforces the cyclical character of the design. Furthermore, above the ceiling structure, a rectangular volume in part of the living room, kitchen and clothes closet is raised 3 feet, which is highlighted as indicated in Figure 9. It is set along an orthogonal axis of $\mathrm{D}_{1}$. Functionally, this accommodates clerestory windows to bring light into the center of the house. (Figure 9). The whole floor plan exhibits only $\mathrm{C}_{1}$ symmetry. It can be said that in the final design, there is an abundance of symmetries within the parts while the strict symmetry of the whole is negated. This approach is outside the classical design lexicon, in which the strict symmetrical disposition of the building parts is emphasized and where the combination of local and global symmetry is the driving force behind the organization of the design. As demonstrated in this analysis, although the plan does not exploit all the possibilities of the subsymmetries of the square, various subsymmetries with rotation and reflection are stratified into a single-story design, extremely rare in architectural design. It appears that Schindler initially set up a symmetrical frame for the project, only to break it, arriving at a final, asymmetrical design that meets all the functional requirements.

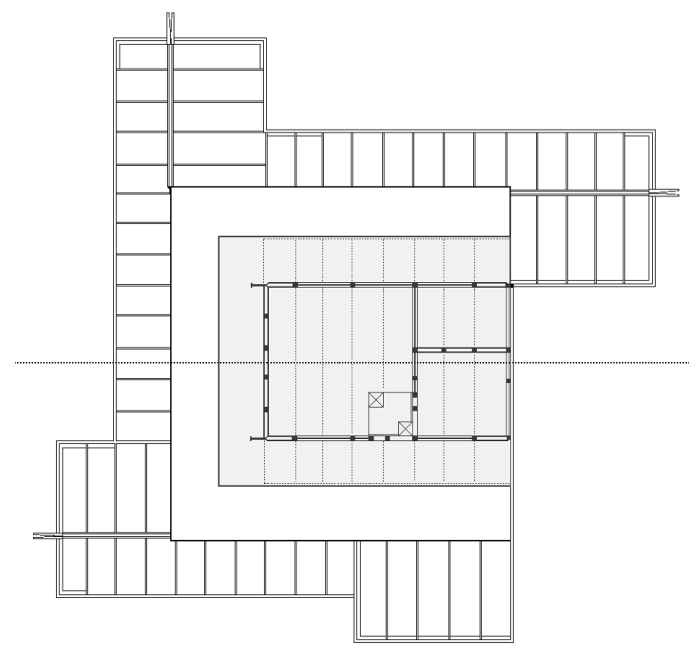

Figure 9. The 3-foot high raised ceiling design: $D_{1}$ subsymmetry along a horizontal orthogonal axis; the roof joists are spaced 24 inches apart on center

\section{After analysis, synthesis}

The underlying reason for analysis is to be able to do synthesis. In this section, we build up a single building design, making use of the symmetries and subsymmetries of a square motif. Then, all the subsymmetries with their hierarchical structure are made evident in individual floors of a new project, including the full symmetry group $\mathrm{D}_{4}$ of the square and the identity. First of all, we need to choose a minimum building element. Essentially we want to provide the clearest possible

92 NEXUS NETWORK JOURNAL - VOL. 3, NO. 1, 2001 


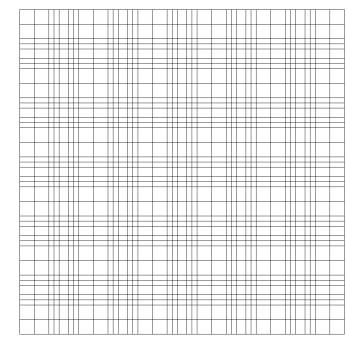

a) A selected grid

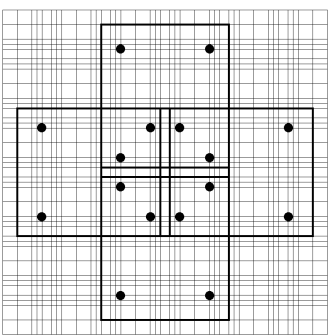

b) D4 (1st Floor)

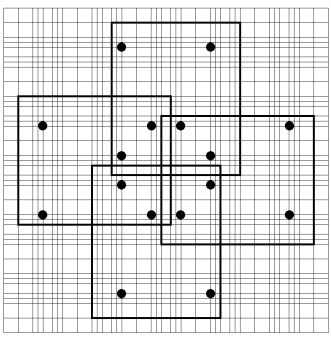

c) C4 (2nd Floor)

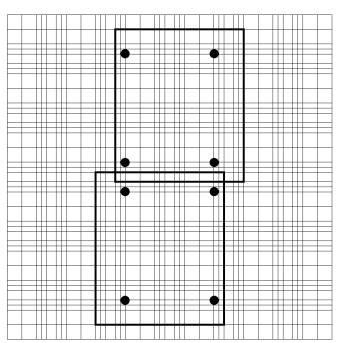

d) C2 (3rd Floor)

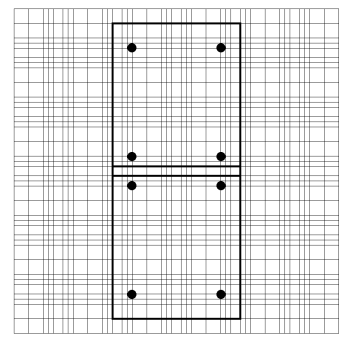

e) D2 (4th Floor)

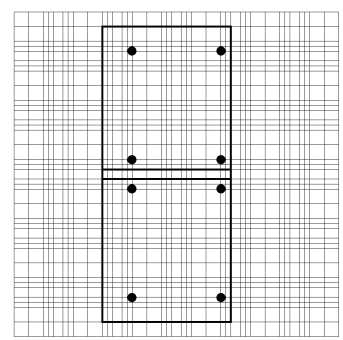

f) D1 (5th Floor)

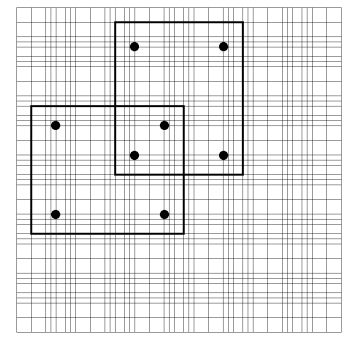

g) D1 (6th Floor)

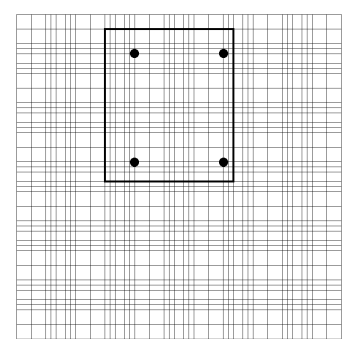

h) $\mathrm{C} 1$ (7th Floor)

Figure 11. The synthetic approach to the design of a new architectural composition, a seven-story building in which each floor presents a distinctive subsymmetry 
picture of the design as a whole. The minimum element is composed of dots representing columns and rectangles representing floors. And then, as was done in the analytic example, we take the subsymmetries of the square with a grid system. The subsymmetries of the square are used as compositional tools; a grid system is used as an underlying parti to juxtapose building elements. Any other regular grids or tessellation can be used but a "dimensional grid" is implemented in our exercise. In his paper "A Class of Grids" [1981], Lionel March defines and catalogues a series of grids where he regulates "three linear elements A, B, C, which had distinct integer dimensions with $\mathrm{a}<\mathrm{b}<\mathrm{c}$. The grids were designed to accept permutations of these elements". Among many distinctive possible composites, the permutation of the 3, 4 and 5 linear elements that produce a polyrhythmic grid, $3+1+1+2+1+1+3$, is chosen (Figure 10).

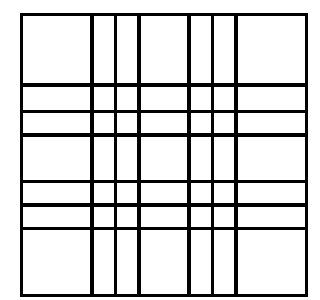

Figure 10. The polyrhythmic pattern derived from the permutation of the 3, 4 and 5 linear elements

For this exercise it is assembled in a $5 \times 5$ array in this exercise (Figure 11a). The square of the grid has $\mathrm{D}_{4}$ symmetry. Placing the element on top of the grid, we arrange columns and floors differently at each level to form symmetric designs illustrating different subgroups or subsymmetries of $\mathrm{D}_{4}$. Each floor level is designed in terms of different subgroup or subsymmetry of the square. Then, we stack the levels up, floor by floor, in a standard height, creating a 3-D schematic building design. We start at the lowest level of the symmetrical order. The first floor consists of the full symmetry of the square, which is $\mathrm{D}_{4}$ symmetry (Figure $11 \mathrm{~b}$ ). Columns and floors are set along the full symmetry of the square with four rotations and four reflections. The second floor illustrates the pinwheel type rotational design characteristic of $\mathrm{C}_{4}$ symmetry (Figure 11c). Column positions are the same as the previous $\mathrm{D}_{4}$ configuration, but the floors are arranged based on the four quarter-turns of $\mathrm{C}_{4}$ symmetry. Compared to level one, floor planes are shifted to maintain rotational symmetry, while destroying the mirror symmetry of the first level. The third level represents the half-turn $\mathrm{C}_{4}$ symmetrical design (Figure 11d). Although columns are set as for $\mathrm{D}_{2}$ symmetry, the two floors are set along the half-turn rotation, breaking the mirror symmetry. The fourth floor incorporates the two mirror reflections, which form the subgroup $\mathrm{D}_{2}$ of $\mathrm{D}_{4}$ (Figure 11e). Columns and floors are set along two mirror axes, which are perpendicular to each other. The fifth floor is composed using a single mirror axis (Figure 11f). The design looks similar to the level below, but upon a closer inspection, we see that columns are set along the $\mathrm{D}_{2}$ orthogonal axis, and floors are shifted from the central axis destroying the vertical mirror axis. The sixth floor illustrates a second $\mathrm{D}_{1}$ symmetry group (Figure $11 \mathrm{~g}$ ). Here the mirror axis is placed at a diagonal to the grid. The top-level floor (seventh floor) has only $360^{\circ}$ rotation as a symmetry, which is denoted as $\mathrm{C}_{1}$ (Figure $11 \mathrm{~h}$ ). Mathematicians would say this level has only the trivial symmetry group. The final design achieved by the synthetic method appears in a threedimensional representation in Figure 12. 


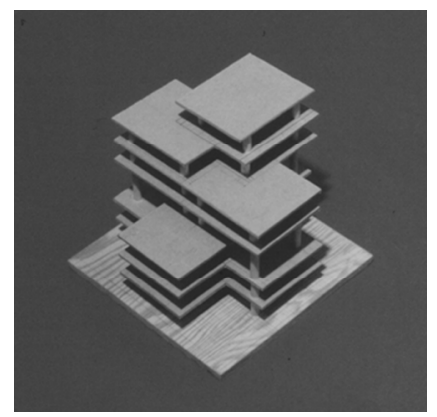

Figure 12. Final design achieved by the synthetic method in a 3-D representation

\section{Conclusion}

We have described and demonstrated methods of analysis and synthesis in architectural composition, based on the algebraic structure of the symmetry groups of a regular polygon. Using this method, the Popenoe House has been analyzed to show a unique application of symmetry operations with regard to the spatial organization. In the design, Schindler explores a variety of subsymmetries superimposed one upon the other around a single central point in an architectural design. A similar geometric order and symmetric technique is applied to the generation of a new design. However, the synthetic process differs a little from the analytic one. Rather than a simple rhythmic grid of the same unit [Klee 1953], we have used a more complex polyrhythmic grid derived from the permutation of three distinctive linear elements. Also, whereas the analytic example shows the superimposition of various subsymmetries in a single floor plan, the synthetic design has a different type of subsymmetry in each floor plan. The synthetic example is an experimental design using the method of symmetry groups to generate one among many other possible designs. Thus we see that symmetry is an effective method for reading the spatial order of complex designs as well as for composing new designs in architecture.

First published in the NNJ Winter 2001

\section{Acknowledgment}

A previous version of this paper was presented at the Bridges 2000 conference in Winfield, Kansas, USA [Park 2000b].

\section{Notes}

1. [Schindler 1946] This article was written in 1944 but published in 1946. Schindler noted that Frederic Heath "spurred him to organize his idea of the unit plan". Schindler had already indicated much earlier in his 1916 "Lecture Note" that he was concerned with a simple unit with its subdivision: "architect to choose his own 'Unit', unit to be subdivided $1 / 2$ and 1/4." See [Schindler 1916]. In this period he was interested in the square unit as well as the rectangle, triangle and circle. He believed that its application depended on "different expression of building". But in his lifelong practice, unlike Frank Lloyd Wright, he never used any geometric forms of unit other than square.

2. In [Schindler 1946], Schindler argued that the architect who wishes to deal with the phenomenon of space has to have not only an innate talent, but also a method that helps to visualize the space forms in his mind, and to improve "his mental image" of the space. The rationale for Schindler's proportional system is that with the system, the forms of space are 
freely conceived and precisely measured in the architect's mind through the process of visualization.

3. In his "Lecture Note" [1916], Schindler wrote about symmetry as the "simplest means of expressing an organism: adjusting two units with one axis", and "relations of two units". To him, symmetry is understood as the harmony that results from the relationship of two units. This simple definition might have exerted a deep influence upon his development of the butterfly patterns of spatial organization.

4. [Park 2000] systematically analyzed the two buildings in terms of their symmetrical hierarchies.

5. [Park 1999] provides a detailed study on symmetric operations in the Schindler Shelter (19331943).

6. No explanation of the circumstances surrounding the architect's intentions for the project is provided by the archives, nor is there any description of the project. For short explanations of the projects, see [Gebhard 1980; Sheine 1998].

7. Gebhard [1980] writes some descriptions on the cabins, saying "Schindler's unwillingness to use the machine to modify the climate and environment worked far better in these vacation houses than his own Hollywood house. All three were located in the dry inner valley between the coast and the central desert, and they were not meant to be used when the weather was either too cold or too hot."

8. The idea of locality is a significant issue in Schindler's "space architecture" in his lifelong practice. In the discussion of the "location", Schindler emphasizes the relationship between "the parts and the whole" where "nothing is alone, everything is connected relationships". See [Schindler 1916]. Subsequently, in "Space Architecture" [Schinder 1934], Schindler also emphasized the significance of the building as "the product of a direct impregnation by the nature of the locale", criticizing Wright's sculptural approach for the Imperial Hotel. Later on, in answer to a questionnaire from the School of Architecture at the University of Southern California (1949), Schindler described the relation of house and lot: "The conventional house is conceived as a solid mass growing out of the ground. The space house as a space form becomes a part of room formed by the lot, the surroundings, contours, and firmament." Schindler's emphasis on the site is not a self-contained manifestation of physical environment. Then, in an unpublished article written in 1952, he emphasized again that "Since a composition in space deals with the out-doors as its raw material, it is obvious that the building should melt down into its surrounding that these define the character of the interior as well"; see [Schindler 1952].

9. A similar parti is found in his early Free Public Library project (1920). The balcony floor plan of the Library project is equally subdivided into the nine squares, where overall spaces are distributed concentrically for functional necessity; see [Park 1996].

15. Schindler's use of pinwheel symmetry in an individual building is earlier than that of Wright. Lionel March states that "Frank Lloyd Wright does not use this symmetry for a parti until St. Mark's Tower of 1929" [March and Steadman 1971]. Yet, Wright used the symmetry much earlier in a housing design, Quadruple Block Plan (1901).

\section{References}

HERMANN, W. 1989. Gottfried Semper: In Search of Architecture. Cambridge, MA: MIT Press. GEBHARD, David. 1993. The Architectural Drawings of R. M. Schindler: The Architectural Drawing Collection. University Art Museum, University of California, Santa Barbara: Garland Publications. 1980. Schindler. Santa Barbara and Salt Lake City: Peregrine Smith. 
Goethe, J.W von. 1946. Versuch die Meamophose der Pflanzen zu Erklaren (Gotha). 1790. A. Arber, trans. Chronica Botanica 10 (2): 63-126.

KLEE, Paul. 1953. Pedagogical Sketchbook. London: Faber and Faber.

MARCH, Lionel. 1981. A Class of Grids. Environment and Planning B: Planning and Design 8: 325-382.

MARCH, Lionel and Philip Steadman. 1971. The Geometry of Environment. London: RIBA Publications Limited.

PARK, Jin-Ho. 2000a. Subsymmetry analysis of architectural design: some examples. Environment and Planning B: Planning and Design 27.

- 2000b. Subsymmetry Analysis and Synthesis of Architectural Designs. Pp. 79-86 in Bridges 2000: Mathematical Connections in Art, Music, and Science, Conference Proceedings 2000, Reza Sarhangi, ed. Winfield, KS: Southwestern College.

1999. "The Architecture of Rudolf Michael Schindler: The Formal Analysis of Unbuilt Work.” Ph.D. dissertation, University of California Los Angeles, 1999.

. 1996. Schindler, Symmetry and the Free Public Library, 1920. Architectural Research Quarterly 2: 72-83.

1995. "A Formal Analysis of R. M. Schindler's Free Public Library Project.” MA Thesis, University of California Los Angeles.

SCHINDLER, Rudolf Michael. 1916. "Lecture Note" (manuscript). The Architectural Drawing Collection, University of California, Santa Barbara.

—. 1934. Space Architecture. Dune Forum (1934): 44-46.

1946. Reference Frames in Space. Architect and Engineer 165.

1952. "Visual Technique" (unpublished essay). The Architectural Drawing Collection, University of California, Santa Barbara.

SheINE, J. 1998. R.M. Schindler. Barcelona: Editorial Gustavo Gill.

WeIL, Hermann. 1952. Symmetry. Princeton: Princeton University Press.

WRIGHT, Frank Lloyd. 1929. "In the Cause of Architecture: Composition as Method in Creation." Unpublished essay.

\section{About the author}

Jin-Ho Park is an assistant professor in the School of Architecture at the University of Hawaii. He currently teaches architectural design studios as well as a series of courses on Design and Computation. He earned his BS in architecture from Inha University, Korea, and his MA and Ph.D. in architecture from UCLA. He is the first recipient of the R.M. Schindler Fellowship of the Beata Inaya Trust Fund, 1996/7, and twice recipient of the Chancellor's Dissertation Fellowship, 1998/99. The focuses of his academic research are on the architecture of R. M. Schindler (unbuilt works), design and computation, including fundamentals of architectonics (proportion, symmetry and compartition), shape grammars, virtual reality and digital media. $\mathrm{He}$ is author of numerous articles in journals such as Architectural Research Quarterly and Environment and Planning B: Planning and Design. 